\title{
Characterization of Six Missense Mutations in the Tissue-Nonspecific Alkaline Phosphatase (TNSALP) Gene in Chinese Children with Hypophosphatasia
}

\author{
Haiou Yang ${ }^{\mathrm{a}, \mathrm{e}}$ Lili Wang ${ }^{\mathrm{b}, \mathrm{e}}$ Juan Genga ${ }^{\mathrm{a}, \mathrm{e}}$ Tingting Yu ${ }^{\mathrm{b}}$ Ru-en Yao ${ }^{\mathrm{b}}$ Yongnian Shen ${ }^{\mathrm{c}}$ \\ Lei Yinc Daming Ying ${ }^{c}$ Rongkui Huang ${ }^{c}$ Yunfang Zhou ${ }^{c}$ Huijin Chen ${ }^{c}$ Lanbo Liuc \\ Xi Moc Yiping Shen ${ }^{c, d}$ Qihua Fu ${ }^{a}$ Yongguo Yu ${ }^{b, c}$ \\ aDepartment of Laboratory Medicine, Shanghai Children's Medical Center, Shanghai Jiaotong University \\ School of Medicine, Shanghai; 'Institute of Pediatric Translational Medicine, Shanghai Children's \\ Medical Center, Shanghai Jiaotong University School of Medicine, Shanghai; 'Department of Internal \\ Medicine, Shanghai Children's Medical Center, Shanghai Jiaotong University School of Medicine, \\ Shanghai; 'Department of Laboratory Medicine, Boston Children's Hospital, Boston, MA; ${ }^{\mathrm{e} T h e s e}$ authors \\ contributed equally to this work, and should be considered as co-first author.
}

\section{Key Words}

Hypophosphatasia • Tissue-nonspecific alkaline phosphatase (TNSALP) gene • Gene mutation analysis • Plasmids • Enzymatic activity

\begin{abstract}
Aims: Hypophosphatasia, a rare inherited disease characterized by defective mineralization of bone and teeth, is caused by various mutations in the tissue-nonspecific isoenzyme of alkaline phosphatase (TNSALP) gene. Our aim was to determine the mutations on TNSALP gene in three Chinese children diagnosed as having hypophosphatasia. Methods: Genomic DNA was extracted from whole blood samples of patients and their parents. The TNSALP coding regions were then sequenced. Plasmids expressing wild-type or various mutants were built and in vitro studies were performed in order to determine whether these amino acid replacements could affect the TNSALP enzymatic activity. Results: Six missense mutations were identified from three independent pedigrees. Of the six missense mutations, four were novel and two had been previously reported. The Y28D, A111T and T389N mutants displayed only negligible ALP activity in vitro compared to the wild-type (WT) TNSALP. The defect was mainly due to the significantly decreased protein expression in the $66 \mathrm{KD}$ immature forms and the nearly undetectable protein expression in the $80 \mathrm{KD}$ mature forms. Moreover, all three mutants
\end{abstract}


had a dominant negative effect on the WT protein when co-transfected with TNSALP (WT). M219V and R136L mutants both exhibited partial enzymatic activities which were consistent with reduced protein expression in both forms of TNSALP which further exhibited moderate dominant-negative effect. In addition, Y388H mutant showed weak ALP activity. Western blot analysis indicated that the extreme reduction in signal from the mature forms of TNSALP could be the main cause of decreased enzymatic activity, since a strong signal was observed in the immature forms. Conclusion: Six missense mutations were identified in three Chinese hypophosphatasia pedigrees with subnormal serum ALP activity. Our results show that the low activity of serum ALP in the three patients is due mainly to a defect in the protein expression of the mutants. This may be the underling molecular mechanism for hypophosphatasia in these patients.

Copyright (C) 2013 S. Karger AG, Basel

\section{Introduction}

Hypophosphatasia is a rare inherited disorder characterized by defective mineralization in bones and teeth accompanied by a deficiency in serum alkaline phosphatase (ALP) activity. The frequency of the severe form of the disease has been estimated to be 1/300,000. The disease is caused by loss-of-function mutations in the tissue-nonspecific alkaline phosphatase (TNSALP) gene [1-5]. As of Apirl 2013, a total of 267 TNSALP mutations have been reported worldwide, the most prevalent of which is a missense mutation, which accounts for 74.5\% of all mutations (http://www.sesep.uvsq.fr/03_hypo_mutations.php). The strong allelic heterogeneity in the TNSALP gene results in clinical heterogeneity. The symptoms of this disease vary widely and are classified into five major categories depending on the age at diagnosis: perinatal, infantile, childhood, adult and odonto forms. Recently, the perinatal form was further divided into two types, lethal and benign [6,7].

The TNSALP protein is synthesized as a $66 \mathrm{KD}$ immature form with mannose-type oligosaccharides and is converted to an $80 \mathrm{KD}$ mature form with complex-type oligosaccharides as it migrates from the endoplasmic reticulum (ER) to the Golgi apparatus and is then localized on the cell surface as a GPI-anchored protein $[6,8,9]$. During synthesis of the enzyme, monomeric precursors spontaneously dimerize and interact selectively with one magnesium and two zinc metal ions as cofactors [10,11]. Mutations in the TNSALP gene can affect any step in protein expression, folding, modification, trafficking or dimerization of TNSALP [12-18]. Furthermore, residual activities of TNSALP mutant proteins can exhibit dominant negative effects leading to mild hypophosphatasia phenotypes in heterozygous carriers [18]. The dominant negative effect of a mutation is characterized by the synthesis of a defective protein monomer that interferes with the wild-type (WT) protein product, when both assemble into the dimeric protein complex and form a heterodimer. This may result in only partial enzyme activity or a nonfunctional enzyme.

In the present study, we sequenced the TNSALP gene in three patients that were diagnosed as having hypophosphatasia. The TNSALP gene was also sequenced in the patients' parents. The following six missense mutations were found [c.82T $>\mathrm{G}$ (p.Y28D), c.331G>A (p.A111T), c.407T>G (p.R136L), c.655A>G (p.M219V), c.1162T >C (p.Y388H), and c.1166C >A (p.T389N)]. Plasmids expressing various mutants were then built in order to determine whether these amino acid replacements could affect the TNSALP enzymatic activity. Using this tool, we found that these mutants exhibited various levels of enzymatic activity in vitro. Moreover, we found that these residual replacements could affect TNSALP protein expression at different steps indicating that residuals in the TNSALP protein have different roles in enzymatic activity. 
Yang et al.: Mutation in TNSALP with Hypophosphatasia

Table 1. Clinical feature of the three pedigrees in this study

\begin{tabular}{|c|c|c|c|c|c|}
\hline Family & Status & Age & $\begin{array}{l}\text { Tooth } \\
\text { loss }\end{array}$ & $\begin{array}{c}\text { Rachitic chest } \\
\text { deformity }\end{array}$ & $\begin{array}{c}\text { Alkaline } \\
\text { phosphatase }\end{array}$ \\
\hline \multirow{3}{*}{1} & Patient & 18 months & + & & $7 \mathrm{IU} / \mathrm{L}(95-500)$ \\
\hline & Father & & & & $51 \mathrm{IU} / \mathrm{L}(40-150)$ \\
\hline & Mother & & & & ND \\
\hline \multirow{3}{*}{2} & Patient & 5.5 years & + & + & $27 \mathrm{IU} / \mathrm{L}(95-500)$ \\
\hline & Father & & & & $23 \mathrm{IU} / \mathrm{L}(40-150)$ \\
\hline & Mother & & & & $17 \mathrm{IU} / \mathrm{L}(40-150)$ \\
\hline \multirow{3}{*}{3} & Patient & 18 months & + & & $38 \mathrm{IU} / \mathrm{L}(95-500)$ \\
\hline & Father & & & & $53 \mathrm{IU} / \mathrm{L}(40-150)$ \\
\hline & Mother & & & & $17 \mathrm{IU} / \mathrm{L}(40-150)$ \\
\hline
\end{tabular}

\section{Materials and Methods}

\section{Case report}

Three pedigrees exhibiting hypophosphatasia were included in the present study. The parents of each patient were asymptomatic and non-consanguineous. The clinical features and biochemical profiles of each patient were consistent with the diagnosis of hypophosphatasia.

The first patient, an 18-month-old female, was referred due to premature loss of deciduous teeth. Biochemistry revealed that her serum activity of alkaline phosphatase (ALP) was negligible (7 IU/l, ageadjusted reference range of $95 \mathrm{IU} / \mathrm{L}-500 \mathrm{IU} / \mathrm{L}$ ) (Table 1). The patient's father had a normal serum ALP level of $51 \mathrm{IU} / \mathrm{L}$ (age-adjusted reference range of $40 \mathrm{IU} / \mathrm{L}$ - $150 \mathrm{IU} / \mathrm{L}$ ) (Table 1). Her parents had no clinical manifestations of hypophosphatasia.

The second patient, a 5.5-year old male, was characterized clinically with rachitic chest deformity, delayed walking and a waddling gait. He also had subnormal serum ALP activity (27 IU/L) upon biochemical laboratory testing (Table 1). His father and mother had moderately decreased serum ALP activities of 23 IU/L and 17 IU/l (Table 1), respectively.

The third patient, an 18-month-old male, was also referred to our hospital for premature loss of deciduous teeth as well as appendicular muscle weakness, especially in both thighs. Low serum ALP activity was noted (38 IU/L) upon testing (Table 1). His father had normal serum ALP levels of $53 \mathrm{IU} / \mathrm{L}$ while his mother had abnormal serum ALP activity (Table 1) (Fig. 1b) with complaints of easy fatigability.

\section{Mutation analysis}

Genomic DNA was extracted from whole blood samples of patients and their parents using a Genomic DNA purification Kit (QIAGEN GmbH, Hilden, Germany). A polymerase chain reaction (PCR) analysis was carried out using 12 sets of specific primers that covered the entire coding region of the TNASLP gene as described previously [19]. For each fragment, PCR was carried out in a total volume of $25 \mu \mathrm{L}$ that contained about $100 \mathrm{ng}$ of genomic DNA, $1 \times$ PCR buffer, $2 \mathrm{mmol} \mathrm{MgCl} 2,0.2 \mathrm{mmol}$ dNTPs, $0.2 \mu \mathrm{mol}$ of each primer, and $1 \mathrm{U}$ Taq DNA polymerase (Takara, Dalian, China). The PCR products were amplified for 35 cycles. Each frag- 


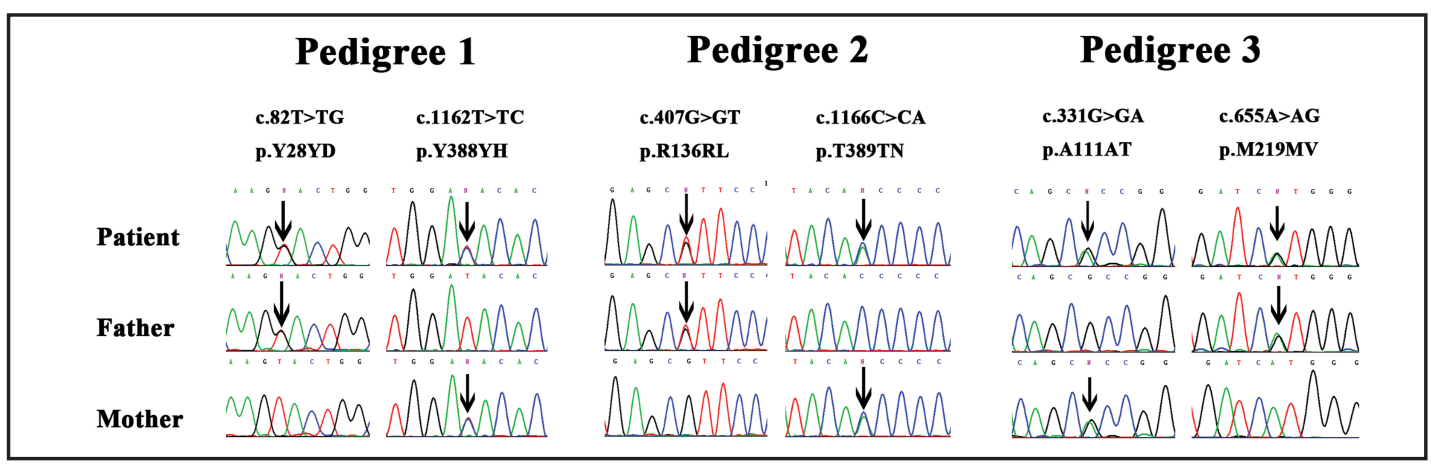

Fig. 1. The sequencing results of the TNSALP gene in the three pedigrees. All of the patients were compound heterozygous carriers and their parents were carriers of heterozygous mutations. Arrows indicated the mutated sites.

ment was denatured at $95^{\circ} \mathrm{C}$ for 30 seconds, annealed at $60^{\circ} \mathrm{C}$ for 30 seconds, and extended at $72^{\circ} \mathrm{C}$ for 50 seconds using an Eppendorf Mastercycler® Pro thermal cycler (Eppendorf, Hamburg, Germany). PCR products were then subjected to DNA sequencing via the ABI 3130XL sequencer (Applied BioSystems, Foster City, CA, US). Each novel mutation was tested via sequencing in at least 100 unrelated normal individuals to ensure that the change was not a frequent polymorphism.

\section{Plasmid construction}

TNSALP proteins were produced using the pEGFP-N1 expression vector and were PCR amplified using the specific primers (data not shown). The PCR products were then successively cloned into the pEGPF-N1 vector with the restriction site provided by the PCR primers: XhoI and EcoRI. The complete construct was verified by sequencing. The pEGFP-N1-TNSALP clone was modified by site-directed mutagenesis in order to establish six TNSALP mutants. Mutagenic primers for the generation of pEGFP-N1-TNSALP-Y28D, A111T, R136L, M219V, Y388H and T389N were designed using the Primer X design program (data not shown). Reactions were performed with the QuikChange II Site-Directed Mutagenesis Kit (Stratagene, The Netherlands) according to the manufacturer's instructions. Mutated plasmid DNA clones were totally sequenced in sense and antisense directions to exclude any further base exchanges, apart from the introduced mutation.

\section{ALP assays}

Plasmids were transiently transfected into HEK 293T cells with LipofectAMINE 2000 reagent (LifeTechnologies, Inc.) using the methodology recommended by the manufacturer. Each assay was duplicated or triplicated in independent experiments. HEK $293 \mathrm{~T}$ cells were plated in 24 -well plates at $1.5 \times 10^{5}$ cells/well in triplicate. At a definite time point, the medium was removed, and cells were washed with cold phosphate-buffered saline (PBS) and lysed with $0.2 \mathrm{ml}$ of lysis buffer (1\% TritonX-100/0.5\% sodium deoxycholate/0.05\% sodium dodecylsulphate) in PBS. A protease inhibitor mixture (Shennengbocai, Shanghai, China) was added to the cell lysate. The cell lysate was incubated for $20 \mathrm{~min}$ at $37^{\circ} \mathrm{C}$ to extract TNSALP. The lysate and media were centrifuged at $15000 \times \mathrm{g}$ for $10 \mathrm{~min}$ at $4^{\circ} \mathrm{C}$ to remove insoluble material. ALP activity was determined using the commercially available kit, Enzyline (ALP alkaline phosphates test reagent kit, Unicel DXC6000, Suzhou, China).

\section{TNSALP protein expression by Western blot analysis}

The remaining cell lysate was prepared in $5 \times$ SDS loading buffer and was directly analyzed by SDSPAGE and transferred onto PVDF membranes. After blocking in 5\% BSA in Tris buffered saline containing 0.1\% Tween-20, the membrane was incubated with anti-TNSALP (Bioworld, Nanjing, China) or anti-GAPDH antibody (Bioworld). The antigen-antibody complex was then immunostained by DyLight ${ }^{\mathrm{TM}}$ 800-conjugated secondary antibodies (KPL, Gaithersburg, MD) and visualized with an Infrared Imaging System (Licor Odyssey, Lincoln, NE, USA). Each experiment was performed at least three times with several cells being analyzed each time. 


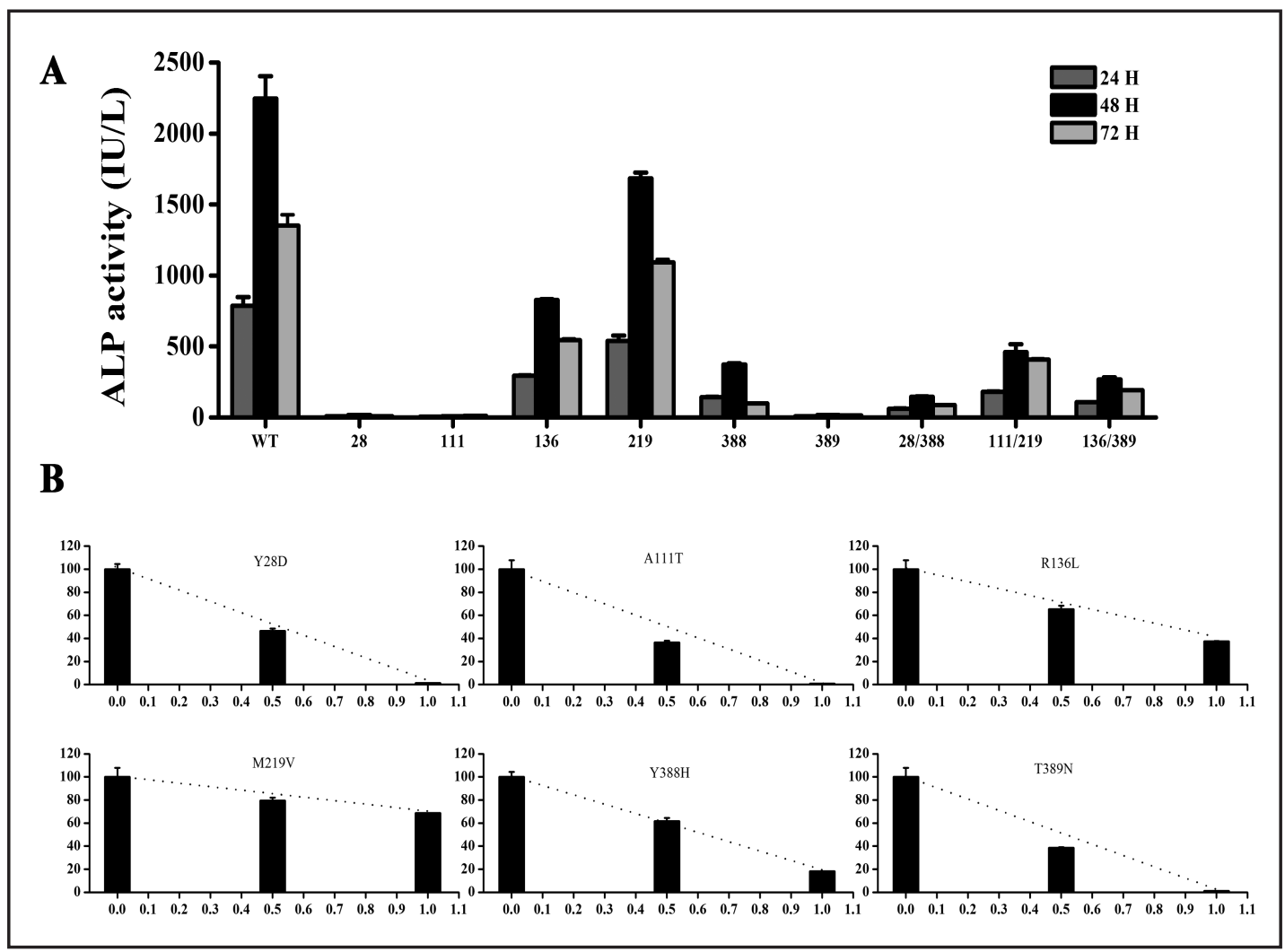

Fig. 2. Enzyme activity of TNSALP mutants in transfected HER 293T cells. (A) Cells that were transiently transfected with plasmids encoding WT, Y28D, A111T, R136L, M219V, Y388H or T389N were homogenized and assayed for ALP activity at the indicated time points. Data are representative of three independent experiments with similar results. (B) Cells were co-transfected with plasmids encoding WT in combination with various mutants in 1:0, 1:1 and 0:1 ratios. At the 24-hour time point, cells were homogenized and assayed for ALP activity (expressed in \% WT, Y axis). Y28D, A111T and T389N exhibited only low ALP while R136L and M219V displayed partial enzymatic activities. All the five mutants showed a dominant negative effect on the WT.

\section{Statistical analysis}

All data are shown as mean \pm SD of triplicate samples. Student's t-test was used for comparison between groups. A p value of $<0.05$ was considered statistically significant.

\section{Results}

\section{Gene mutation analysis}

As hypophasphatasia is mainly caused by different mutations in the TNSALP gene, we screened the entire coding region of TNSALP in the three pedigrees. Nucleotide sequence analysis showed that all three patients were compound heterozygous carriers [Patient 1 : c.82T > TG (p.Y28YD), c.1162T>TC (p.Y388YH), patient 2: c.407G>GT (p.R136RL), c.1166C>CA (p.T389TN) and patient 3: c.331G>GA (p.A111AT), c.655A>AG (p.M219MV)] (Figs. 1a, b and c). We found that the six missense mutations present in the three patients were all inherited from their parents. The results also showed that all six adults were carriers of a heterozygous missense mutation [the father of patient 1: c.82T>TG (p.Y28YD), the mother of patient 1: c.1162T>TC (p.Y388YH), the father of patient 2: c.407T >TG (p.R136RL), the mother of patient 2: c.1166C >CA (p.T389TN), the father of patient 3: c.655A >AG (p.M219MV), and the mother of patient 3: c.331G>GA (p.A111AT)] (Figs. 1a, b and c). According to the Human Gene 


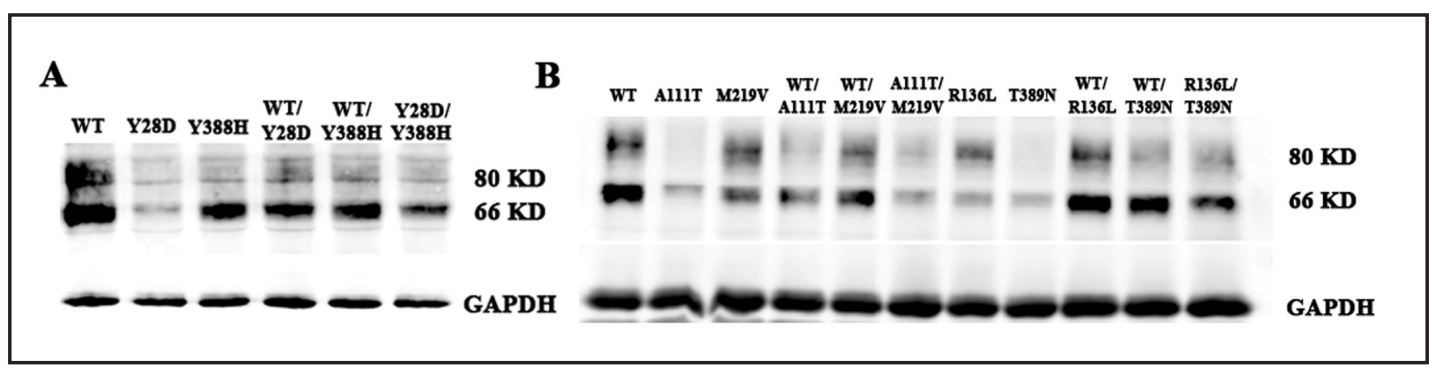

Fig. 3. Transient expression of TNSALP mutant proteins in HER 293T cells. Cells were transiently transfected with plasmids encoding WT, Y28D, A111T, R136L, M219V, Y388H or T389N, and were homogenized 72 hours later. Lysates were analyzed for TNSALP expression by Western blot; GAPDH was used as the loading control. Data shown is representative of three separate experiments.

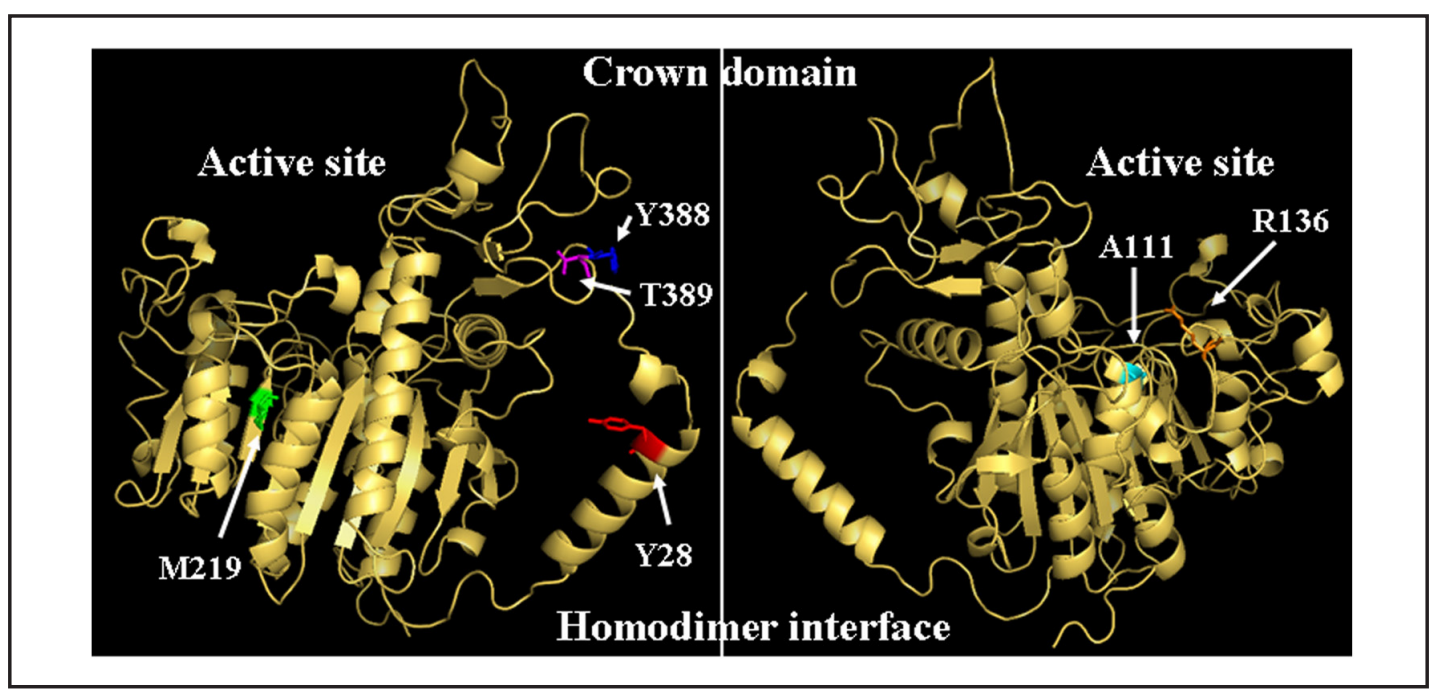

Fig. 4. Localization of the affected residues by 3D structural modeling. A Ribbon presentation of the WT TNSALP monomer is shown. Y28 is located at the $\alpha$-helix which is involved in the homodimer interface. A111 and R136 are located at the active site domain. M219 is located in the $\beta$-sheets, which contribute to the stability of the hydrophobic region. Y388 and T389 in the crown are highlighted.

Mutation Database, the 1000 Genomes and the Exome Variant Server database, four of the six mutations [c.82T > G (p.Y28D), c.407T > G (p.R136L), c.655A > G (p.M219V) and c.1166C >A (p.T389N)] were novel while the others had been previously reported. Three bioinformatics tools (SIFT, PolyPhen-2 and Mutation taster) (http://sift.jcvi.org, http://www.mutationtaster.org, and http://genetics.bwh.harvard.edu/pph2/index.shtml) were also used to evaluate the impact of these missense mutations on the function of the TNSALP protein. These indicated that the six missense mutations could substantially disrupt the normal function of TNSALP (data not shown).

\section{Enzymatic activity of mutant TNSALP}

In order to define the molecular properties of six mutants, HEK 293T cells were transiently transfected with a pEGFP-N1 vector encoded with WT or mutant TNSALP. At a definitive time point, HEK $293 \mathrm{~T}$ cells were homogenized and assayed for ALP activity. In contrast to the WT TNSALP, the Y28D, A111T, and T389N exhibited negligible activity (Fig. 2a), displayed only $1.72 \%$ (Y28D), $0.83 \%$ (A111T) and 1.27\% (T389N) of WT in 24 hours, $0.70 \%$ (Y28D), $0.42 \%(\mathrm{~A} 111 \mathrm{~T})$ and $0.82 \%(\mathrm{~T} 389 \mathrm{~N})$ of WT in 48 hours, $0.22 \%$ (Y28D), $1.07 \%$ (A111T) and 
$1.21 \%$ (T389N) of WT in 72 hours respectively. R136L and Y388H also had very low ALP activity, whereas M219V displayed only moderately decreased ALP activity. The activity of the WT was slightly compromised when co-expressed with Y28D, A111T, R136L, M219V and T389N, indicating a moderate dominant negative effect of these five mutants (Fig. 2b). However, Y388H did not exhibit any significant dominant negative effects (Fig. 2b). These results indicate that all residue replacements can affect the catalytic function of TNSALP. Y28D, A111T, R136L, M219V and T389N residue replacements abrogated the catalytic function of TNSALP and demonstrated a considerable inhibitory impact on WT activity.

\section{Protein expression of the mutant TNSALP}

To further explore whether the observed decrease in the ALP activity of mutants was due to impaired TNSALP protein expression, HEK 293T cells transiently expressing either WT, Y28D, A111T, R136L, M219V, Y388H or T389N were lysed and analyzed by Western blot after being cultured for 72 hours. As expected, the WT exhibited the strongest signals in both the $66 \mathrm{KD}$ immature and the $80 \mathrm{KD}$ mature forms, due to posttranslational protein modifications (Figs. 3a and 3b). However, consistent with the ALP activity, Y28D, A111T, and T389N showed significantly decreased protein expression in the $66 \mathrm{KD}$ form and an undetectable signal in the $80 \mathrm{KD}$ form. R136L and M219V displayed both forms of TNSALP and the expression levels were partially affected due to the amino acid substitution. These results indicate that these residue replacements can affect the TNSALP protein expression, especially Y28D, A111T, and T389N. Furthermore, Y388H exhibited only the $60 \mathrm{KD}$ band, which was slightly lower than the WT, and exhibited only a negligible level of the $80 \mathrm{KD}$ band. This data suggests that the $\mathrm{Y} 388 \mathrm{H}$ mutant may affect TNSALP posttranslational protein modifications and that variable missense mutants could affect the enzymatic activity of TNSALP at different steps of protein expression.

\section{Discussion}

Hypophosphatasia is a monogenic disease that is mainly caused by various mutations in the TNSALP gene. Currently, at least 267 distinct mutations and 16 polymorphisms are known to correlate with hypophosphatasia. The most prevailing mutation type is the missense mutation which accounts for $74.5 \%$ of all mutations [1-4]. Some TNSALP missense mutations had been previously tested for their impact on the enzymatic activity of TNSALP by transient transfection experiments using COS1/7 or HEK 293T cells. It was reported that the mutations p.A115V, p.A162T, p.D277A and p.F310L had residual TNSALP enzyme activity and were properly anchored on the membrane [20-23]. However, other mutations, including p.R54C, p.N153D, p.E218G, p.D289V, p.G317D and c.1559delT, did not exhibit any residual ALP activity, had defects in membrane-anchoring and seemed to accumulate in the $\mathrm{RE} /$ Golgi apparatus before degradation in the proteasome [21, 24-28]. Kiminitsu Oda et al. demonstrated that the p.A116T protein failed to fold properly and forms disulfide-bonded aggregates, though it was indeed capable of interacting with the WT and reaching the cell surface [18]. Furthermore, Beck et al. revealed that the p.G63V and p.D378V mutants were concentrated in the subcellular region and had a dominant-negative effect on the WT TNSALP protein $[15,29,30]$. According to the structural features of the TNSALP protein, done with a 3D model, the TNSALP missense mutations associated with hypophosphatasia are classified as follows: 1) active site or active site vicinity, 2) active site valley, 3) homodimer interface, 4) crown domain, 5) calcium site or calcium site vicinity and 6) others [10]. Previous studies suggest that a mutation leading to varying degrees of diminished activity does not necessarily affect a functional domain, but could instead, affect the structural conformation of the TNSALP molecule [14].

In the current study, we identified six missense mutations in three patients, from three different Chinese families, who were diagnosed as having hypophosphatasia. The three patients presented with defective bone or teeth mineralization and low serum ALP activity. 
Nucleotide sequencing analysis revealed that the three patients were compound heterozygous and the mutations were inherited from their heterozygous parents. In order to determine the functional impact of these missense mutations on the properties of TNSALP, we conducted a series of in vitro experiments through construction of mutant as well as WT TNSALP-expressing plasmids via site-directed mutagenesis and transiently transfected HEK 293T cells. All six mutants exhibited variable levels of residual TNSALP enzymatic activity, particularly, p.Y28D, p.A111T and p.T389N, which displayed only negligible catalytic activity in vitro. Consistent with the reduced enzymatic activity, the protein expression of all six mutants was also significantly diminished in HEK 293T cells. Analysis of the 3D structural model showed that Y28 is located in the $\alpha$-helix domain, which is involved in the homodimer interface, while A111 is located at the $\alpha$-helix that is in the active site or active site vicinity (Figs. 4a and 4b). In addition, T389 is located at the crown domain. All of the three residue replacements lead to missing signals in the western blot analysis of the $80 \mathrm{KD}$ protein, suggesting that the three mutations may directly or indirectly affect the function of TNSALP, either due to diminished catalytic activity or the instability of proteins prone to degradation before migrating to the cell membrane. In both the $66 \mathrm{KD}$ and $80 \mathrm{KD}$ forms, the protein signal intensities of the R136L and M219V mutants were observed, but were moderately diminished when compared to WT TNSALP suggesting that these mutants are still able to form dimers, undergo glycosylation, and properly anchor to cell membranes. In the 3D structure modeling, R136 is located at the surface of the active site and could be involved in substrate approach and stabilization (Fig. 4b). M219 is located in a $\beta$-sheet and its residue is buried in the TNSALP hydrophobic region, which contributes to the floor of the active site cleft. This phenomenon is similar to one found in an earlier study of missense mutations (p.V217A/K) found in the same $\beta$-sheet which may cause instability or influence the calcium-binding vicinity [13]. Y388 is adjacent to T389 and is located at the same crown domain (Fig. 4a). However, in the Y388 mutation, the $66 \mathrm{KD}$ immature form could be observed while the $80 \mathrm{KD}$ mature form was absent, suggesting that the amino acid substitution from Tyr to His in the 388 residue site may disrupt the local structural stability, lead to conformational alteration, and in turn, affect posttranslational protein modifications.

Hypophosphatasia is highly variable in its clinical symptoms and signs, which can range from stillbirth with no mineralized bone to early loss of teeth in late adulthood, but with no bone symptoms [6,7]. The severe form of hypophosphatasia can result in rachitic chest deformity and death in children. Currently there is no cure for this rare disease; however, several attempts have recently been made to development therapies to alleviate the symptoms of this disorder. An enzyme replacement therapy using a soluble human recombinant TNSALP with a bone-targeted deca-aspartate motif shows promise and clinical trials are underway [31]. Early diagnosis and treatment can effectively prevent progression of this disease and improve patient quality of life. Genetic tests are currently used to diagnose hypophosphatasia, however to correlating genotypes and phenotypes still requires a lot of effort as most patients have compound heterozygosity of TNSALP genes, making it difficult to determine the respective roles of each mutation.

In conclusion, six missense mutations were identified in three Chinese hypophosphatasia pedigrees with subnormal serum ALP activity. Using site-directed mutagenesis and transfection studies, in combination with 3D structural modeling, we evaluated the functional impact of these six mutations on ALP activity in vitro. Our results show that the low activity of serum ALP in the three patients is due mainly to a defect in the protein expression of the mutants. This may be the underling molecular mechanism for hypophosphatasia in these patients.

\section{Conflicts of Interest}

The authors declare no conflict of interest. 
Yang et al.: Mutation in TNSALP with Hypophosphatasia

\section{Acknowledgements}

This study is further supported by Shanghai Pujiang Talent Program (No.13PJ1406200, to YGY) from Shanghai Municipal Science and Technology Commission, and supported by Shanghai Municipal Health Bureau "New 100 Talents Program" (No.XBR2011046, to QHF), and the grant from Shanghai Science \& Technology Commission Major Project (No. 11dz1950300, to YGY and YG-2011-MS28). We thank all of the members of these families for their participation.

\section{References}

1 Harris H: The human alkaline phosphatases: What we know and what we don't know. Clin Chim Acta 1990;186:133-150.

2 Whyte MP: Physiological role of alkaline phosphatase explored in hypophosphatasia. Ann N Y Acad Sci 2010;1192:190-200.

3 Whyte MP: Hypophosphatasia and the role of alkaline phosphatase in skeletal mineralization. Endocr Rev 1994;15:439-461.

4 Mornet E: Hypophosphatasia: The mutations in the tissue-nonspecific alkaline phosphatase gene. Hum Mutat 2000;15:309-315.

-5 Mornet E, Yvard A, Taillandier A, Fauvert D, Simon-Bouy B: A molecular-based estimation of the prevalence of hypophosphatasia in the european population. Ann Hum Genet 2011;75:439-445.

6 Mornet E: Hypophosphatasia. Orphanet J Rare Dis 2007;2:40.

7 Zurutuza L, Muller F, Gibrat J, Taillandier A, Simon-Bouy B, Serre L, Mornet E: Correlations of genotype and phenotype in hypophosphatasia. Hum Mol Genet 1999;8:1039-1046.

$>8$ Weiss MJ, Ray K, Henthorn P, Lamb B, Kadesch T, Harris H: Structure of the human liver/bone/kidney alkaline phosphatase gene. J Biol Chem 1988;263:12002-12010.

-9 Weiss MJ, Cole D, Ray K, Whyte MP, Lafferty MA, Mulivor RA, Harris H: A missense mutation in the human liver/bone/kidney alkaline phosphatase gene causing a lethal form of hypophosphatasia. Proc Natl Acad Sci USA 1988;85:7666-7669.

10 Mornet E, Stura E, Lia-Baldini AS, Stigbrand T, Ménez A, Le Du MH: Structural evidence for a functional role of human tissue nonspecific alkaline phosphatase in bone mineralization. J Biol Chem 2001;276:3117131178.

11 Harada T, Koyama I, Matsunaga T, Kikuno A, Kasahara T, Hassimoto M, Alpers DH, Komoda T: Characterization of structural and catalytic differences in rat intestinal alkaline phosphatase isozymes. FEBS J 2005;272:2477-2486.

12 Lia-Baldini AS, Brun-Heath I, Carrion C, Simon-Bouy B, Serre J, Nunes M, Mornet E: A new mechanism of dominance in hypophosphatasia: The mutated protein can disturb the cell localization of the wild-type protein. Hum Genet 2008;123:429-432.

13 Chang KC, Lin PH, Su YN, Peng SSF, Lee NC, Chou HC, Chen CY, Hsieh WS, Tsao PN: Novel heterozygous tissue-nonspecific alkaline phosphatase (tnap) gene mutations causing lethal perinatal hypophosphatasia. J Bone Miner Metab 2012;30:109-113.

14 Brun-Heath I, Lia-Baldini AS, Maillard S, Taillandier A, Utsch B, Nunes ME, Serre JL, Mornet E: Delayed transport of tissue-nonspecific alkaline phosphatase with missense mutations causing hypophosphatasia. Eur J Med Genet 2007;50:367-378.

15 Mentrup B, Marschall C, Barvencik F, Amling M, Plendl H, Jakob F, Beck C: Functional characterization of a novel mutation localized in the start codon of the tissue-nonspecific alkaline phosphatase gene. Bone 2011;48:1401-1408.

16 Makita S, Al-Shawafi HA, Sultana S, Sohda M, Nomura S, Oda K: A dimerization defect caused by a glycine substitution at position 420 by serine in tissue-nonspecific alkaline phosphatase associated with perinatal hypophosphatasia. FEBS J 2012;279:4327-4337. 
Yang et al.: Mutation in TNSALP with Hypophosphatasia

17 Numa N, Ishida Y, Nasu M, Sohda M, Misumi Y, Noda T, Oda K: Molecular basis of perinatal hypophosphatasia with tissue-nonspecific alkaline phosphatase bearing a conservative replacement of valine by alanine at position 406. FEBS J 2008;275:2727-2737.

18 Ishida Y, Komaru K, Oda K: Molecular characterization of tissue-nonspecific alkaline phosphatase with an ala to thr substitution at position 116 associated with dominantly inherited hypophosphatasia. Biochim Biophys Acta 2011;1812:326-332.

19 Orimo H, Hayashi Z, Watanabe A, Hirayama T, Hirayama T: Novel missense and frameshift mutations in the tissue-nonspecific alkaline phosphatase gene in a japanese patient with hypophosphatasia. Hum Mol Genet 1994;3:1683-1684.

-20 Cai G, Michigami T, Yamamoto T, Yasui N, Satomura K, Yamagata M, Shima M, Nakajima S, Mushiake S, Okada S: Analysis of localization of mutated tissue-nonspecific alkaline phosphatase proteins associated with neonatal hypophosphatasia using green fluorescent protein chimeras. J Clin Endocrinol Metab 1998;83:3936-3942.

21 Fukushi-Irie M, Ito M, Amaya Y, Amizuka N, Ozawa H, Omura S, Ikehara Y, Oda K: Possible interference between tissue-non-specific alkaline phosphatase with an arg54--> cys substitution and acounterpart with an asp277--> ala substitution found in a compound heterozygote associated with severe hypophosphatasia. Biochem J 2000;348:633.

22 Shibata H, Fukushi M, Igarashi A, Misumi Y, Ikehara Y, Ohashi Y, Oda K: Defective intracellular transport of tissue-nonspecific alkaline phosphatase with an ala162--> thr mutation associated with lethal hypophosphatasia. J Biochem (Tokyo) 1998;123:968-977.

23 Watanabe H, Takinami H, Goseki-Sone M, Orimo H, Hamatani R, Ishikawa I: Characterization of the mutant (a115v) tissue-nonspecific alkaline phosphatase gene from adult-type hypophosphatasia. Biochem Biophys Res Commun 2005;327:124-129.

24 Fukushi M, Amizuka N, Hoshi K, Ozawa H, Kumagai H, Omura S, Misumi Y, Ikehara Y, Oda K: Intracellular retention and degradation of tissue-nonspecific alkaline phosphatase with a gly317--> asp substitution associated with lethal hypophosphatasia. Biochem Biophys Res Commun 1998;246:613-618.

25 Ishida Y, Komaru K, Ito M, Amaya Y, Kohno S, Oda K: Tissue-nonspecific alkaline phosphatase with an asp289--> val mutation fails to reach the cell surface and undergoes proteasome-mediated degradation. J Biochem (Tokyo) 2003;134:63-70.

-26 Ito M, Amizuka N, Ozawa H, Oda K: Retention at the cis-golgi and delayed degradation of tissue-nonspecific alkaline phosphatase with an asn153--> asp substitution, a cause of perinatal hypophosphatasia. Biochem J 2002;361:473.

27 Watanabe A, Karasugi T, Sawai H, Naing BT, Ikegawa S, Orimo H, Shimada T: Prevalence of c. 1559delt in alpl, a common mutation resulting in the perinatal (lethal) form of hypophosphatasia in japanese and effects of the mutation on heterozygous carriers. J Hum Genet 2010;56:166-168.

28 Komaru K, Ishida Y, Amaya Y, Goseki-Sone M, Orimo H, Oda K: Novel aggregate formation of a frame-shift mutant protein of tissue-nonspecific alkaline phosphatase is ascribed to three cysteine residues in the cterminal extension. FEBS J 2005;272:1704-1717.

29 Müller HL, Yamazaki M, Michigami T, Kageyama T, Schönau E, Schneider P, Ozono K: Asp361val mutant of alkaline phosphatase found in patients with dominantly inherited hypophosphatasia inhibits the activity of the wild-type enzyme. J Clin Endocrinol Metab 2000;85:743-747.

-30 Lia-Baldini A, Muller F, Taillandier A, Gibrat J, Mouchard M, Robin B, Simon-Bouy B, Serre J, Aylsworth A, Bieth E: A molecular approach to dominance in hypophosphatasia. Hum Genet 2001;109:99-108.

-31 Whyte MP, Greenberg CR, Salman NJ, Bober MB, McAlister WH, Wenkert D, Van Sickle BJ, Simmons JH, Edgar TS, Bauer ML: Enzyme-replacement therapy in life-threatening hypophosphatasia. N Engl J Med 2012;366:904-913. 